\title{
Epidemiology of non-alcoholic and alcoholic fatty liver diseases
}

\author{
Souveek Mitra ${ }^{1}$, Arka De ${ }^{2}$, Abhijit Chowdhury ${ }^{1,3}$ \\ ${ }^{1}$ Indian Institute of Liver and Digestive Sciences Sitala (east), Jagadishpur, Sonarpur, Kolkata, India; ${ }^{2}$ Department of Hepatology, Post Graduate \\ Institute of Medical Education and Research Chandigarh, Chandigarh, India; ${ }^{3}$ Department of Hepatology School of Digestive and Liver Diseases \\ Institute of Post Graduate Medical Education \& Research Kolkata, India \\ Contributions: (I) Conception and design: All authors; (II) Administrative support: None; (III) Provision of study materials or patients: All authors; \\ (IV) Collection and assembly of data: All authors; (V) Data analysis and interpretation: All authors; (VI) Manuscript writing: All authors; (VII) Final \\ approval of manuscript: All authors. \\ Correspondence to: Abhijit Chowdhury. Indian Institute of Liver and Digestive Sciences Sitala (east), Jagadishpur, Sonarpur, 24 pgs(s), Kolkata 700150 , \\ India. Email: achowdhury2002@yahoo.co.in.
}

\begin{abstract}
Liver diseases are fast emerging as global health priorities. Fatty liver is described in the setting of non-alcoholic fatty liver disease (NAFLD) as well as alcoholic liver disease (ALD), the pathogenesis of excess fat being different in the two conditions while both are important components of the changing face of burden of liver diseases worldwide. They are intimately associated with a globalized economy and an increasingly homogenous socio- cultural order with a westernized lifestyle. The accompanying adoption of a progressively sedentary life, consumption of diet dense in calories facilitate development of NAFLD while a spiraling upward trend in alcohol use along with earlier age of drinking as well as increased amount of per capita alcohol consumption increases the prevalence of ALD globally. Adverse health outcomes in NAFLD as well as ALD are caused not only by progressive liver fibrosis that is the most significant factor for liver related and all-cause mortality in both but also by non-liver (cardiovascular, cancer, accidents, neurological) clinical outcomes that calls for a multidisciplinary and social approach to these conditions. We present here an outline of facets of epidemiology of both NAFLD as well as ALD along with its' public health implications. A broad-based integrated approach that incorporates social, behavioral as well as biological targets need to be undertaken at a health system level in a planned manner for these evolving liver health priorities that disproportionately challenges the low- and middle-income countries of Asia, South America and Africa.
\end{abstract}

Keywords: Lean; obesity; steatosis

Received: 19 June 2019; Accepted: 05 September 2019; Published: 05 April 2020.

doi: $10.21037 / \operatorname{tgh} .2019 .09 .08$

View this article at: http://dx.doi.org/10.21037/tgh.2019.09.08

Accumulation of excessive fat in the liver is the common denominator underlying the two most common and emerging causes of chronic liver disease, alcoholic liver disease (ALD) and non-alcoholic fatty liver disease (NAFLD), that are emerging public health issues globally. The burden of both ALD and NAFLD are increasing worldwide $(1,2)$. ALD occur as a component of a broader perspective of alcohol abuse disorders, is frequently associated with psychiatric comorbidities and is the most frequent cause of morbidity, health care utilization and mortality in alcohol use disorders (3-6). This is in contrast to NAFLD that occurs as an essential component of metabolic disorders that are associated with insulin resistance as the pathophysiological hallmark and is clinically manifest as hepatic, pancreatic, cardiac endothelial cell dysfunction and disease. In NAFLD, death is most commonly due to cardiovascular disease and often nonhepatic cancers apart from liver disease $(7,8)$. The current global march of NAFLD as a public health challenge parallels the global upsurge for food intake, increase in per capita income, sedentary lifestyle, increasing body mass index and finally is an expression of an excess of caloric 
intake over expenditure by an individual (9-14).

While hepatocytes swell with excess fat, primarily as triglycerides, in both the conditions and set the grounds for inflammation and most importantly, fibrosis-the pathogenesis of fatty liver and subsequent progression of liver disease is different in ALD and NAFLD. An expanded, dysfunctional, insulin resistant adipose tissue with ectopic fat deposition and hepatic storage due to an imbalance is the hallmark of NAFLD with lipotoxicity as the primary driver of hepatocyte injury (15-17). In ALD, on the other hand, the accumulated fat participates as a rather innocent bystander in an inflammatory process that injures fat laden hepatocytes starting with resident and nonresident liver infiltrating immune cells as the trigger $(18,19)$. The epidemiology including genetic risk factors for ALD and NAFLD are different, the only connect between them being broadly the habits, lifestyle, socio economic as well as cultural factors in the population that serve as facilitator $(14,20)$. An intriguing feature is that the two often coexist in view of the shared lifestyle context and the fact that both are so common that they frequently coexist by chance alone. This coexistence creates a compound etiological framework for progressive liver disease and a synergistic dualism that poses an emerging public health burden across the globe. ALD existed through centuries and is one of the earliest identified condition causing chronic liver diseases, afflicting the poor and the affluent in the society with almost similar propensity. NAFLD, on the other hand, is a "new world" phenomenon, is a biological marker of social affluence and sedentary lifestyle and disproportionately influence the public health issues of well off and transitional economies $(1,7,8)$. It is important to note, as discussed below, that these countries with transition from a low to middle income category provide the best match bedrock for the socio- cultural milieu that favors both NAFLD as well as ALD, stressing the existing beleaguered health systems in these regions with a healthcare priority that need as much attention to behavioral changes as to the biology of the disease (8,21-23).

\section{Epidemiology of NAFLD}

NAFLD is an umbrella term for the liver diseases that are characterized primarily by storage of excess macro vesicular fat ( $>5 \%$ of the hepatocytes) because of a perturbation of the homeostatic mechanisms that regulate synthesis versus utilization of fat in the liver $(7,15,16)$. Diagnosis of NAFLD currently needs an exclusion of a history of more than moderate alcohol intake and absence of drug exposure as well as defined genetic disorders that can culminate in a similar phenotype of fatty liver (24). This "negative" definition of NAFLD underscore the fact that we still lack a specific biological marker that could precisely characterize the condition, setting it aside from similar pathologies that differ in pathogenesis as well as outcome (25). A subset of individuals with NAFLD develop progressive liver disease, marked by hepatocyte injury (ballooning), inflammation and finally, fibrosis, an entity designated as non-alcoholic steatohepatitis (NASH) $(24,25)$. Presence and the grade of Fibrosis are most important prognostic determinant in NAFLD and NASH is classically considered to be the phenotype of NAFLD that underlie development of progressive liver disease, particularly fibrosis and therefore, is clinically relevant (26-29). However, clinical, epidemiological and therapeutic intervention studies have brought forth the primacy of excess fat storage, not only as the "soil" but also in view of the fact that fat itself may progress to fibrosis with minimal evident inflammation-a condition coined as "Non-alcoholic steatohepatitis" (30).

The public health importance of NAFLD stems from its' multifaceted impact on morbidity, mortality and health care utilization globally $(8,21,25)$. NAFLD and particularly, NASH fibrosis is associated with an excess allcause mortality and also liver related mortality in general population. NASH is an important cause of chronic liver disease, cirrhosis of the liver (often included in the cryptogenic basket) and hepatocellular carcinoma, often developing in non-cirrhotic livers (31-33) too. NASH is also an important underlying etiology for acute on chronic liver failure (ACLF). NASH is the fastest emerging cause of liver transplantation in the United states, UK and also in the developing countries $(25,32)$. Intriguingly however, most deaths in NAFLD are due to cardiovascular disease while overall cancer mortality is also increased in NAFLD (31). The excess cardiovascular risk in fatty liver has been reported even in populations with relatively low background adiposity as measured by BMI, indicating a biological link between the insulin resistance (IR) is the cellular abnormality that underlies the diverse disorders that are popularly placed under the term metabolic syndrome (MS) (34). Chronic indolent inflammation associated with MS, an entity often called "meta inflammation", connects the different non-communicable diseases (NCD) together. NAFLD is therefore the hepatic expression of a systemic disorder in which aberrant insulin action and resultant altered metabolic flux cause underlie inflammation that span 
multiple tissues and organ systems. NAFLD epidemiology is intricately linked with the changing burden and epidemiology of NCD s that is currently underway globally (35).

One of the important caveats of NAFLD epidemiology is non-availability of a disease-specific biomarker that is powerful and simple for large scale population-based studies. The clinically relevant subset of NAFLD, NASH and fibrosis, are mostly histologically defined entities and therefore, are difficult to estimate in the epidemiological context (36-39). Non-invasive serum markers, used singly or in panels, as well as various imaging modalities, particularly magnetic resonance (MR), has been used in select studies for an assessment of the burden of clinically relevant NAFLD (38-40). However, serum markers still lack uniformity, standardization across populations and are yet to receive wide acceptance in epidemiological studies although they are promising. MRI and transient elastography based methods can measure fat and liver fibrosis with fair precision and reproducibility but are difficult to use in large scale epidemiological setting in view of the cost, limited availability of equipment and for MR, lack of expertise. As a result, most of the available epidemiological data on NAFLD are ultrasound based that detect liver fat in a semi quantitative manner. In addition, ALT as a surrogate of liver injury, has also been used in some studies despite its' nonspecific nature in view of its' simplicity and wide availability. In general, studies requiring abnormal liver blood tests to make the diagnosis of NAFLD have reported much lower prevalence estimates compared with studies that use imaging methods $(36,41)$.

\section{Prevalence}

Pooled prevalence of NAFLD globally is $25.24 \%$ with wide geographical variation across the world. Highest prevalence rates-mostly ultrasound based-has been reported from Middle East and South American countries (around 30\%) whereas the limited number of studies from Africa reports a much lower prevalence $(13 \%)(10,36,42)$. Majority of the studies on NAFLD epidemiology, however, has been from the USA and North America with a NAFLD prevalence rate of $21-24.7 \%$. Studies using serum markers consistently recorded a lower prevalence compared to those based on imaging. Similar NAFLD prevalence has been reported from Europe (24\%) in a meta-analysis of studies published till 2015 (10). In sheer numbers of people needing clinical attention, NAFLD poses a significant public health burden in USA and Europe (64 million and 52 million respectively) while Asia, by virtue of its larger and expanding population, a rapidly urbanizing society with an upwardly mobile economic drift and a NAFLD burden that is increasing faster than the developed countries of the West, is going to bear the brunt of the global NAFLD burden in the coming years (43). The vast geographical spread of Asia, the regional diversity in terms of economic development, diet and lifestyle amongst different countries and also within countries along with possible genetic influences are factors that account for wide variation of NAFLD prevalence among Asian areas (43-51). Thus, it varies from $12.5-38 \%$ in Chinese Mainland, 23-26\% in Japan, 27\% in Korea, $12-51 \%$ in Taiwan, $28 \%$ in Hongkong, 9-32\% in India and $5-30 \%$ in other areas of South Asia and far East (Srilanka, Malayasia, Srilanka and Indonesia). An additional aspect of epidemiology of NAFLD in Asia is the so called "lean NAFLD" or "Non-obese NAFLD" that constitutes around $10 \%$ of the NAFLD subjects and was initially described in Asian populations, indicating the remarkable propensity of Asians to develop MS linked clinical conditions at anthropometric parameters that are considered sub threshold for obesity. NAFLD in so called "lean" subjects also owe its' genesis to an expanded adipose tissue that defies classical metrics but follow a similar pattern of clinical outcomes $(52,53)$.

\section{Incidence}

Estimates of incidence of NAFLD (new onset NAFLD in people who did not have it earlier over a defined period of time) are even sparse in view of the longitudinal nature of such studies as well as inherent difficulty of non-availability of a non-invasive biomarker that would detect NAFLD when used repetitively, reproductively with precision and ease of use in large scale population available studies are heterogeneous in terms of methods and data (54-58). In a study that followed 11,448 subjects for 5 years, incidence of NAFLD documented by ultrasound was $12 \%(n=51,418)$. A study from Israel reported an incidence rate of 28 per 1,000 person-years. The most meticulously performed study in this respect had been in an Asian population in Hongkong and used MR-spectroscopy that measures fat with maximum accuracy, reporting an incidence of $13.5 \%$ over a period of 3-5 years. In an analysis of 237 studies from Asia (18 studies included for incidence analysis), the pooled annual NAFLD incidence rate was 50.9 cases per 1,000 person-years (95\% CI: 44.8-57.4). In patients with NAFLD, the annual incidence of hepatocellular carcinoma was 
1.8 cases per $1,000 \mathrm{~d}$ person years (95\% CI: $0.8-3.1)$ and overall mortality rate was 5.3 deaths per 1,000 person-years (95\% CI: 1.5-11.4). Another analytical study on the burden and trends of NAFLD globally observed that the pooled regional NAFLD incidence rate estimates for Asia and Israel were 52.34 per 1,000 (95\% CI: 28.31-96.77) and 28.01 per 1,000 person-years (95\% CI: $19.34-40.57$ ) respectively. Similar studies are available in western population also although using different methodologies. A study based on liver enzymes as a surrogate in a US population under 45 years reported annual rate of increase of ALT elevations from $2.3 \%$ to $4.2 \%$ (almost $90 \%$ increase). In an ultrasound based follow up study over 8.6 years from Italy, the incidence of NAFLD was 18.5 years 1,000 person years.

\section{Changing trends}

NAFLD prevalence is showing a fairly steady upslope in most of the populations over time in the last two decades. A meta regression identified an association between year of study publication and an increase in NAFLD prevalence globally (15\% in 2005 to $25 \%$ in 2010) (10). Compared with the western countries, the increase in NAFLD prevalence over time is more marked in Asia and the Pacific countries reflecting rapid lifestyle changes that are dramatic in these regions $(43,44)$. The epidemiological trends of NAFLD parallels the changes in prevalence of obesity, diabetes and other NCDs that accompany this social transition. The overall food supply of the planet has increased and, despite regional disparities, many parts of the world no longer suffer from starvation and famine. Studies from Japan and China recorded a twofold increase in the prevalence of NAFLD over a decade. Similar trends are noted in other Asian areas also. The prevalence increased from $18.7 \%$ to $27.3 \%$ in South Korea (over the period from 2006 to 2013), increased from $12.9 \%$ to $43.3 \%$ in Shanghai, China (2003 to 2016), increased from $11.5 \%$ to $27.0 \%$ in Taiwan (2006 to 2007 ) and increased from $7.9 \%$ to $51.0 \%$ in Indonesia (2013 to 2015). As mentioned previously, an analysis of 237 studies (13,044,518 participants) on NAFLD epidemiology from Asia noted that NAFLD prevalence increased significantly over time [25.28\% (22.42-28.37\%)] between 1999 and 2005, 28.46\% [26.70-30.29] between 2006 and 2011, and 33.90\% (31.74-36.12\%) between 2012 and 2017 (12-14,45). A study using data from the U.S. National Health and Nutrition Examination Survey found that NAFLD prevalence doubled between the survey periods of 1988 to 1994 and 2005 to 2008 . In general, the reported prevalence of NAFLD in the United States was already around $30-40 \%$ in 2004 and has not increased substantially there after (10).

\section{Epidemiological risk factors for NAFLD}

\section{Obesity, socioeconomic changes and lifestyle}

NAFLD subjects of are almost always obese and obese people have increased prevalence of NAFLD, underscoring the tight pathogenic connection between them. The entire spectrum of obesity, ranging from overweight to obese and severely obese, is associated with NAFLD. In this context, the majority ( $>95 \%$ ) of patients with severe obesity undergoing bariatric surgery will have NAFLD (8,59-61). This relationship is preserved even in the lean or non-obese NAFLD subjects have subtle indicators that fail to be captured by BMI in view of a different pattern of compartmentalization of body fat in them (52). This brings obesity in the center of NAFLD epidemiology that largely parallels the prevalence and determinants underlying the obesity pandemic that is sweeping globally. It is pertinent and important to consider NAFLD epidemiology as an integral component of a broader spectrum of metabolic disorders wherein excessive adipose tissue that is dysfunctional and inflamed sets the grounds for health outcomes that manifests differentially in different organ systems. Body mass index (BMI) is the most widely used surrogate of obesity had generally shown an upward trend globally over the past decades, with the slope of the increase being most steep in the low- and middle-income countries, mostly from Asia and Africa $(62,63)$. NAFLD prevalence follows almost the similar trends with Asian countries bearing the brunt of the emerging burden of NAFLD. While biological factors including genetic predisposition, are important in the present spiraling emergence of obesity and NAFLD as one of its' accompanying clinical correlates, the present upsurge has its' roots primarily in the availability of excess food calories and economic development that is taking place globally. Adoption of a sedentary lifestyle, lack of physical activity, increasing engagement in knowledge based economic work also contribute to increasing prevalence obesity and MS linked clinical conditions. Economic globalization has also a lingering cultural impact with changes in dietary habits with increasing consumption of refined sugars, foods and additives that add calories. It is important that these changes are still evolving and the 
lifestyle-MS interface has multiple micro and intermediate variables including epigenetic events that translate social changes into biological phenomenon.

\section{Diabetes}

Insulin resistance of varying degree is considered to be the critical cellular abnormality that underlie both NAFLD and type 2 diabetes. In this context, a strong association between NAFLD and type 2 diabetes mellitus (T2DM) has been shown, as $>70 \%$ of patients with T2DM have NAFLD. Presence of insulin resistance and diabetes is considered a risk factor for more severe liver disease in NAFLD even in patients with normal levels of serum ALT. Diabetes not only is a frequent co morbidity of NAFLD, it is one of the determinants of downhill progression of the natural history of NAFLD with more inflammation (NASH), accelerated progression of liver fibrosis as well as development of hepatocellular carcinoma (64-68). The burden of NAFLD, with clinically relevant fibrosis affecting up to $20 \%$ of those with both NAFLD and T2DM, seems to be enormous considering the huge number of patients with T2DM worldwide. While type 2 diabetes predisposes to NAFLD, the reverse is also observed. Ultrasonography-defined NAFLD is associated with a twofold to fivefold increased risk of developing T2DM after correction for various lifestyle and metabolic confounders. Importantly, resolution of fatty liver as assessed by ultrasonography resulted in a substantial reduction in the risk of T2DM development, to a level similar to individuals without NAFLD. In this study, individuals with worsening fatty liver over 5 years showed a marked increase in T2DM NAFLD $(69,70)$. Overall, the relationship between Diabetes and NAFLD is much tighter than an association alone, indicate shared pathophysiology and as a clinical condition have a temporal course in which either can predate before co existing.

\section{$A G E$}

NAFLD and aging are strongly correlated and increasing age is one of the most robust epidemiological factors for NAFLD, NASH and fibrosis (48,71-78). Older age is not only a risk factor for hepatic steatosis, but also individuals with older age have a greater likelihood of mortality and disease progression to fibrosis and hepatocellular carcinoma. It is possible that the relationship between age and fibrosis progression may be attributable to the longer duration of disease in the elderly patients with NAFLD.
Most epidemiological studies could find a steady increase in NASH and fibrosis with age, particularly after the fifth decade of life.

\section{Gender}

The influence of sex on NAFLD prevalence and course, however, is not so straightforward. Available studies are diametrically divided between either sex, some demonstrating a female while others a male preponderance in prevalence of NAFLD (79).

\section{$R A C E$}

Racial differences in prevalence of NAFLD have been most evident in the studies from USA that involved multiethnic populations. Hispanic Americans have the highest prevalence of NAFLD followed by Americans of European descent and African Americans having the lowest prevalence (80-82). Ethnic differences are also noted within South America, with Brazil reporting the highest NAFLD prevalence and Peru the lowest The lower prevalence of NAFLD among African Americans than Hispanics despite higher prevalence of obesity amongst Non-Hispanics brings into focus the complexities in pathogenesis of NAFLD implicating the role of genetics as well as epigenetic influences operating through diet, lifestyle and other environmental factors. It is also important to recognize that intra ethnic differences exist particularly amongst Hispanics according to their country of origin-with Mexicans in one study having much higher prevalence of NAFLD compared Dominican or those from Puerto Rico even after correction all other variables (age, sex, IR, obesity, lipids etc.) (83).

\section{Pediatric and adolescent NAFLD including perinatal conditioning}

It is now largely considered that the prevalence of obesity and NAFLD in children as well as adolescents is increasing fast, not only in the western population but also in Asia (84). The differential impact of weight gain during school-years carries a higher risk of NAFLD than weight gain in late adulthood. In a longitudinal study in Europe, a weight increase during childhood an early adolescence was related to all histological features of adult NAFLD, even after adjusting for initial as well as attained BMI $(85,86)$. Among children with similar attained BMIs at 13 years of age, the risk of cirrhosis in adulthood was increased by $16 \%$ 
per 1 unit gain in BMI score at every age from 7 through 13 years. Similarly, weight gain during late adolescence is able to induce an increased susceptibility to developing NAFLD later in life. In the Cardiovascular Risk in Young Finns Study, after a follow up of 31 years, adult NAFLD was predicted by modifiable as well as non-modifiable risk factors during childhood, including BMI and plasma insulin levels, male sex, genetic background (that is, PNPLA3 and TM6SF2 variants) and low birth weight, an emerging risk factor for adulthood NAFLD (87).

Evidence shows that perinatal exposure to environmental challenges may dramatically impact health trajectories in later life. Early life factors such as pre pregnancy obesity, maternal obesity, and abnormal gestational weight gain have been associated with increased rates of obesity in the offspring (87). One of the most extensive longitudinal surveys of pregnancy and childhood, the Raine study enrolled 2,979 women by week 18 of gestation from the antenatal clinic in Perth, and in the past 27 years a total of 2,868 children from this cohort have been followed up at birth and every few years thereafter. After adjusting for adolescent obesity, maternal pre pregnancy obesity and gestational weight gain $>6 \mathrm{~kg}$ by week 18 independently predicted NAFLD in adolescent girls, whereas lower socioeconomic status at birth was the only independent predictor of NAFLD in adolescent boys $(88,89)$.

\section{Lean NAFLD}

Lean NAFLD constitutes from 7-20\% of NAFLD subjects in different studies. Initially described from Asia and considered as a "third world phenotype", this subset of NAFLD has since been described in other populations including Europe and the USA $(52,53,90-96)$. Thus, in Korea, $12.6 \%$ of 29,994 people presenting for general medical examination had ultrasonography diagnosed NAFLD, whereas $27 \%$ of 5,878 non-obese $(<25)$ subjects had NAFLD in another study. A comparatively lower prevalence was reported from China (7\%) and from a rural Indian population $(6.5 \%$ lean, $75 \%$ of $8.7 \%$ overall prevalence of NAFL) in which both ultrasonography followed by CT scan were used for diagnosis of NAFLD. On the other hand, a proton magnetic spectroscopy-based study from Hongkong reported a 19\% prevalence of fatty liver in non-obese subjects in contrast to $61 \%$ in people with higher BMI (40). Representative studies from the West include an analysis of non-obese individuals included in the multiethnic population of NHANSIII (1988 to 1994) demonstrated a NAFLD prevalence of $7.9 \%$ in the USA whereas an ultrasound-based study from Italy showed a $16 \%$ prevalence of lean NAFLD.

Well-designed prospective study on the incidence of lean NAFLD is scarce. In a population-based study that included 565 adults from Hongkong with repeat proton magnetic spectroscopy at mean 47 months, $71 \%$ of individuals were lean at baseline $(<23)$ (55). 7.9\% had developed incident fatty liver at follow up and this was associated with an increasing BMI as well as waist circumference and triglycerides-indicating worsening of anthropometricmetabolic status over time in Lean NAFLD while still remaining within the range of $\mathrm{BMI}$ that is sub threshold for obesity. Similar data has been reported from China (8.8\% in 5-year follow up of on ultrasound) and India (cumulative 5 years incidence of ultrasound NAFLD-31\%) (97). In general, these suggest a $3-5 \%$ annual incidence rates for lean NAFLD and this is mostly associated with expansion of fat mass with increasing adiposity.

\section{Epidemiology of ALD}

\section{The global burden and changing trends of alcobol use}

Globally, alcohol use disorder is the most common substance use disorder with more than 1 million cases and 99 million disability-adjusted life years (DALY) attributed to alcohol use in 2016 (20). After smoking and hypertension, alcohol is the commonest preventable cause of death. Even more concerning is the loss of productive life years as mortality attributable to alcohol is more common in the young and middle-aged population (15-49 years), an age group in which it is the most common cause of DALYs (98). As per World Health Organization (WHO) estimates, 2.3 billion people were actively consuming alcohol in 2016. Alarmingly, the per-capita consumption of alcohol has increased from 5.5 litres in 2000 to 6.4 litres in 2016. Though the percentage of drinkers has decreased in Africa and America, it continues to rise in the WesternPacific, Europe and South-East Asian regions including India and China (99). The demographic pattern of alcohol drinking is also changing with commencement of drinking at a younger age and increased consumption in women. More than 200 diseases and a range of injuries have a link with the use of alcohol including cardiovascular diseases, cirrhosis and several cancers. However, the alcohol attributable fraction is largest for liver diseases particularly cirrhosis (99). 


\section{Alcobol-a significant contributor to the global liver disease burden}

Alcohol is one of the commonest causes of end-stage liver disease with $50 \%$ of cirrhosis related mortality being attributed directly or indirectly to alcohol (1). Worldwide, ALD per-se accounts for $4 \%$ of mortality and $5 \%$ of DALYs with Europe being the worst affected. In 2010, almost half a million deaths were attributable to alcohol related cirrhosis (5). One in ten deaths attributable to alcohol is due to alcohol related cirrhosis and almost 50\% of alcohol attributable mortality is due to liver disease. This amounts to an annual loss of 22 million DALYs $(5,99)$. The prevalence of ALD is approximately $2 \%$ in the US general population with an estimated mortality of 5.5 per 100,000 in 2010 (11,100). In the European Union, 41\% of liver related mortality is attributable to alcohol (101). In India, alcohol is the commonest cause of cirrhosis (34.3\%) and almost $20 \%$ of all liver disease patients (irrespective of etiology) are current alcohol consumers (102). Thus, alcohol amounts for a significant part of the liver disease burden across the world. Further, a significant proportion of liver-related mortality of unknown etiology may well be attributable to alcohol as patients may withhold the history of alcohol use and doctors may not mention alcohol use in the death certificates because of various socio-cultural and insurance-related issues (103). Moreover, many studies have considered ALD in only those patients without other etiologies of liver disease. Concomitant significant alcohol intake may be present in well over $50 \%$ of patients with other liver diseases including Hepatitis C, NAFLD and hemochromatosis $(3,104)$. It is speculated that the burden of ALD related mortality is under-estimated by almost twofold because of these various factors (20).

\section{Economic impact of alcohol use}

While net alcohol consumption is higher in the wealthier countries, the morbidity and mortality associated with its use is more in the economically poorer countries. This geographic variation in the alcohol-attributable disease burden was confirmed in a systematic analysis of the Global Burden of Disease Study 2016 with the burden being higher in countries with low and middle socio-demographic indices (20). Contribution to the economy via employment generation and taxation has often touted by the alcohol industry to thwart policies aimed at curbing alcohol consumption. The fallacy of this argument can be illustrated by taking the European Union as an example, where the estimated economic impact of harmful alcohol use ( $€ 125$ billion) is more than 13 times higher than the reported contribution of the alcohol industry to its economy ( €9 billion) $(105,106)$.

\section{Spectrum of ALD}

ALD includes a spectrum ranging from fatty liver, steatohepatitis and cirrhosis with its complications. Steatosis is seen in $90 \%$ of heavy drinkers and is usually considered innocuous because of its asymptomatic nature and reversibility with abstinence. Nonetheless, it represents a metabolic stress that increases the risk of more severe forms of liver disease and patients with steatosis have decreased long-term survival compared to abstinent controls (107). Among patients with steatosis, approximately $35 \%$ progress to steatohepatitis and $10 \%$ develop cirrhosis (108). Thus, despite the definite epidemiologic link between alcohol and liver disease, a relative minority of alcohol consumers develop clinically significant liver disease and earlier stages are reversible with abstinence.

A more granular insight into the natural history of ALD is provided by a recent systematic review of 37 studies including more than 7,500 individuals with histologically proven ALD. $15 \%$ of hazardous drinkers had normal histology while $27 \%, 24 \%$ and $26 \%$ had fatty liver, steatohepatitis and cirrhosis, respectively. Annual rate of progression to cirrhosis was $1 \%$ in those with normal histology compared to $3 \%$ and $8 \%$ in those with steatosis and fibrosis, respectively. The study also confirmed that steatosis was not completely benign with an annual mortality of $6 \%$ though this was predominantly due to nonliver related causes (109). Alcoholic steatohepatitis (ASH) deserves special mention as this entity is associated with high short-term mortality ( $40-50 \%$ at 6 months) that has largely remained unchanged over the past four decades (110). The exact incidence of ASH is difficult to estimate as biopsy is the gold standard for diagnosis. Most previous studies have used diagnostic coding which is inherently less accurate. Estimates suggest that the incidence of ASH is increasing in both males and females $(111,112)$. In 2010, ASH accounted for $0.83 \%$ of admissions in the U.S (113).

\section{Risk factors for ALD}

\section{Alcobol (amount, duration, type, pattern of intake) and diet}

Among the various factors that influence the risk of developing ALD, the most important are the amount and 
duration of alcohol intake. Early French studies suggested $80 \mathrm{~g} /$ day to be the cirrhogenic dose of alcohol though subsequent studies have shown a much lower threshold particularly in women $(114,115)$. Community based studies from northern Italy demonstrated a linear correlation between alcohol dose and risk of liver disease and cirrhosis (116). Pooled data from various epidemiological studies show that daily consumption of at least $30 \mathrm{gm} /$ day of alcohol for women and $50 \mathrm{gm} /$ day for men for a duration of 5 years can lead to clinically significant liver disease (117). While a dose-effect relationship between alcohol consumption and liver disease has been clearly demonstrated, there is no solid evidence behind a safe limit of alcohol consumption. The type of alcohol intake also influences the risk of ALD with lower risk in wine-only drinkers $(118,119)$. However, it is unclear whether this is due to the protective effect of wine per-se or other confounding life-style factors. Daily consumption without meals doubles the risk of cirrhosis as demonstrated in the recent UK Million Women Study (28). Whether binge drinking entails additional risk is controversial with recent studies yielding conflicting results. Daily drinking conferred the highest risk of cirrhosis in the Danish Cancer, Diet, and Health study cohort (120). However, in a Finnish population-based study of 6366 subjects without baseline liver disease, weekly binge drinking was associated with an increased risk of ALD (HR 6.82 in women and 2.34 in women) and this association was independent of average daily consumption (121). Type of diet also possibly influences the risk of ALD and low-calorie diets rich in iron and polyunsaturated fatty acids have been associated with more florid inflammation in animal models (122).

\section{Gender}

Although, the incidence of alcohol related cirrhosis is higher in males than females $(0.2 \%$ vs. $0.03 \%$ annually), the relative risk of ALD is higher in women for any given amount of alcohol intake (116). This greater vulnerability among women has been traditionally explained by the lower volume of distribution for alcohol with resultant higher blood alcohol levels. Recent evidence suggests that estrogen increases gut permeability with consequent up-regulation of endotoxin receptors in Kupffer cells and higher levels of tumour necrosis factor (123).

\section{Chronic viral hepatitis}

Concomitant Hepatitis C has a synergistic effect with alcohol leading to increased apoptotic cell death, release of damage-associated molecular patterns and proinflammatory cytokines resulting in accelerated fibrosis. The effect of concomitant Hepatitis B infection however remains unclear with conflicting evidence of both a protective and deleterious effect in various studies (124-126). Metabolic comorbidities

Presence of metabolic co-morbidities like diabetes and obesity also influence the risk of ALD. The main risk factors for fatty liver, alcohol and MS, are currently linked to distinct entities, namely ALD and NAFLD. The frequent co-existence of these risk factors has led some authors to suggest the term "BASH" or both alcoholic and NASH to indicate fatty liver disease occurring in patients with metabolic risk factors and alcohol use below the defined thresholds for NAFLD $(127,128)$.

Several previous cross-sectional studies had suggested a protective effect of light-moderate alcohol consumption on NAFLD (129-139) (Table 1). However, a meta-regression analysis of more than 42,000 patients from 6 studies suggested the presence of confounders, particularly lower body weight in patients with modest alcohol consumption (140). More recently, longitudinal studies have shown worsening of histology and non-invasive markers in NAFLD with modest alcohol consumption $(141,142)$ (Table 1). Conversely, MS has also been shown to increase the severity of alcohol related cirrhosis (143). Obesity was associated with increased severity and short-term mortality in alcoholrelated acute-on-chronic liver failure patients in a recent analysis of the APASL-ACLF research consortium (AARC) data base (144).

\section{Genetic and epigenetic factors}

It remains unclear why only $10 \%$ of patients with excessive alcohol consumption develop cirrhosis. Genetic and epigenetic factors have been suggested. A detailed discussion of these factors is beyond the scope of this review. In this respect, polymorphisms of patatain-like phospholipase domain-containing protein 3 (PNPLA3) is the most extensively studied with the $148 \mathrm{M}$ variant having been shown to confer increased risk (145).

\section{HCC in ALD: is surveillance justified?}

Alcohol related hepatocellular carcinoma accounted for 80,600 deaths and 2.14 million DALYs in 2010 (5). Like other etiologies, alcohol related cirrhosis too is a risk factor 
Table 1 Studies exploring the epidemiologic link between alcohol consumption and NAFLD

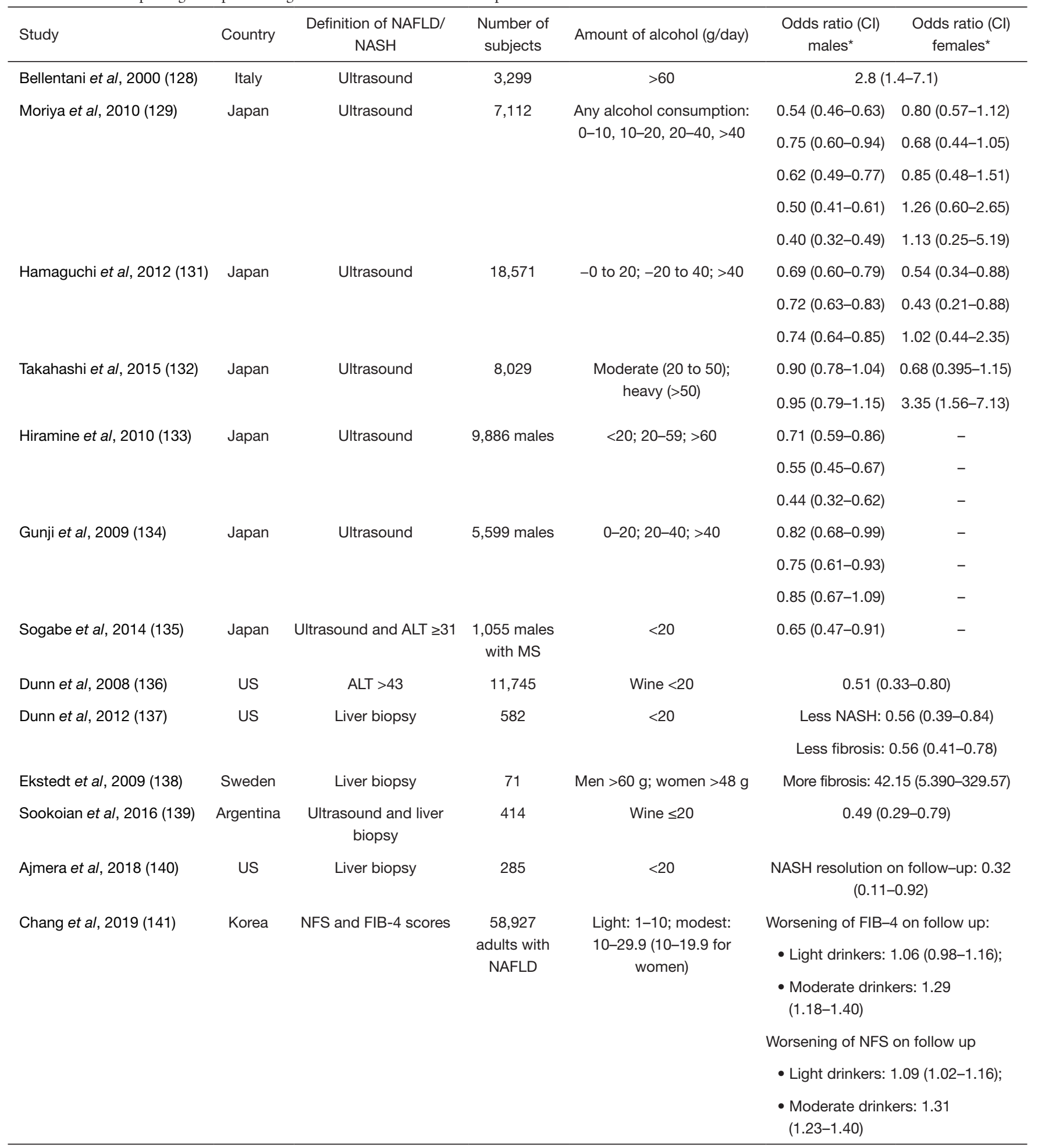

*, outcomes with statistically significant $\mathrm{p}$ values are in bold. NAFLD, non-alcoholic fatty liver disease; NASH, non-alcoholic steatohepatitis; NFS, NAFLD fibrosis score; FIB-4, fibrosis-4 index. 
Table 2 AUDIT-C questionnaire

\begin{tabular}{|c|c|c|c|c|c|}
\hline Question & \multicolumn{5}{|c|}{ Score } \\
\hline $\begin{array}{l}\text { How often do you have a drink containing } \\
\text { alcohol? }\end{array}$ & Never & Monthly or less & $\begin{array}{l}2-4 \text { times a } \\
\text { month }\end{array}$ & 2-3 times per week & $\geq 4$ times a week \\
\hline $\begin{array}{l}\text { How many drinks containing alcohol do you have } \\
\text { on at typical day when you are drinking? }\end{array}$ & $1-2$ & $3-4$ & $5-6$ & $7-9$ & $\geq 10$ \\
\hline
\end{tabular}

Maximum score: 12 ; score of $\geq 4$ in men and $>2$ in women screens "risky drinking".

for development of hepatocellular carcinoma (HCC). However, the incidence is lower than that in cirrhosis due to chronic viral hepatitis (56). In fact, a population-based Danish study questioned the cost-effectiveness of HCC screening in ALD cirrhosis as the annual incidence (1\%) was less than that of the AASLD suggested threshold of $1.5 \%$ per year (57). However, in a recent, large, prospective study from France and Belgium (CIRRAL cohort), the annual incidence of HCC in ALD cirrhosis was 2.9 per 100 patient-years, thereby confirming the cost effectiveness of surveillance in these patients (146).

\section{Changing paradigms of liver transplant in ALD}

ALD is the second commonest indication for liver transplant (LT) in USA and Europe $(147,148)$. LT outcomes in ALD is comparable to that of other etiologies and better than that for Hepatitis C (149). Traditionally 6-month abstinence has been considered a pre-requisite for LT in ALD. However, this mandatory period of abstinence does not correlate with risk of rescividism (150). This is particularly relevant in ASH which is associated with a 6-month mortality of $40-50 \%$. There is mounting evidence regarding the benefit and safety of early LT in steroid non-responsive severe alcoholic hepatitis without increase in recidivism rates (151-153).

\section{Prevention is better than cure}

The WHO envisions a reduction in mortality from ALD cirrhosis to less than 3.2 per 100,000 population by the year 2020. As of now this target seems far away. Moreover, the reduction in mortality in ALD cirrhosis has lagged behind other etiologies (30\% vs. 47\%) with limited evidence for the benefit of therapeutic interventions barring abstinence and LT (154). Thus, the adage "prevention is better than cure" holds true for ALD. The dose-dependent toxicity of alcohol use exists not only at the individual level but also at the population level with national alcohol consumption trends correlating with national liver-related mortality statistics. However, the alcohol industry is a powerful lobby and alcohol is a considerable source of revenue in both developed and developing countries. There is sufficient evidence that simple, cost-effective policy decisions can significantly decrease overall and liver-related mortality attributable to alcohol. These include a systematic increase in taxation, minimum unit price, advertising restrictions, marketing regulations, low-level interventions from clinicians, awareness campaigns, labelled health warnings and protection of children from alcohol and alcoholpromoting advertisements (155).

Abstinence at any time in the natural history of the disease decreases the risk of progression and complications. Moreover, early stages of ALD can revert with abstinence. Thus, screening for harmful alcohol intake should be done at primary contact with the patient. While the alcohol use disorders inventory test (AUDIT) questionnaire is the gold-standard, use of mobile apps and shorter, validated questionnaires like AUDIT-C may be less cumbersome in routine outpatient settings (66) (Table 2). For screening, the National Institute on Alcohol Abuse and Alcoholism (NIAAA) recommends using a single question "How many times in the past year have you had 5 or more drinks in a day (for men) or 4 or more drinks in a day (for women)?" (156). The complete AUDIT should be performed in patients with a positive response (156). Screening for ALD should be done in patients deemed high-risk like those in alcohol rehabilitation centres or patients identified to have harmful drinking.

It also needs to be highlighted that while there are 
partially evidence-based limits for "unsafe" daily or weekly alcohol consumption, there is little evidence behind any proscribed "safe" limit. The apparent health benefits of small quantities of alcohol has been much touted in the medical and non-medical literature. Strong evidence to the contrary has emerged from the Global Burden of Diseases, Injuries, and Risk Factors Study (GBD). There are no safe limits for alcohol as the estimated beneficial effects on cardiac disease are strongly off-set by other health hazards particularly cancer. This notion of "no safe limit of alcohol consumption" has been highlighted in a recent UK guideline $(157,158)$. The evidence-based advice in clinics should thus be "complete abstinence" although this may not be acceptable or feasible for patients due to sociocultural reasons. In such circumstances, intake should be limited to $\leq 2$ drinks/day in females and $\leq 3$ drinks/day in males with each drink being containing 10 grams of alcohol (158).

\section{Acknowledgments}

None.

\section{Footnote}

Conflicts of Interest: The authors have no conflicts of interest to declare.

Ethical Statement: The authors are accountable for all aspects of the work in ensuring that questions related to the accuracy or integrity of any part of the work are appropriately investigated and resolved.

\section{References}

1. Asrani SK, Devarbhavi H, Eaton J, et al. Burden of liver diseases in the world. J Hepatol 2019;70:151-71.

2. Mokdad AH, Forouzanfar MH, Daoud F, et al. Global burden of diseases, injuries, and risk factors for young people's health during 1990-2013: a systematic analysis for the Global Burden of Disease Study 2013. Lancet 2016;387:2383-401.

3. Rehm J, Mathers C, Popova S, et al. Global burden of disease and injury and economic cost attributable to alcohol use and alcohol-use disorders. Lancet 2009;373:2223-33.

4. WHO. Global status report on alcohol 2004. Geneva: World Health Organization, 2004.

5. Rehm J, Samokhvalov AV, Shield KD. Global burden of alcoholic liver diseases. J Hepatol 2013;59:160-8.

6. WHO. Global status report on alcohol and health 2014. Geneva: World Health Organization, 2014.

7. Cohen JC, Horton JD, Hobbs HH. Human Fatty Liver Disease: Old Questions and New Insights. Science 2011;332:1519-23.

8. Rinella M, Charlton M. The globalization of nonalcoholic fatty liver disease: prevalence and impact on world health. Hepatology 2016;64:19-22.

9. Chowdhury A, Younossi ZM. Global Epidemiology and Risk Factors for Nonalcoholic Fatty Liver Disease. In: Chalasani N, Szabo G (eds). Alcoholic and Non-Alcoholic Fatty Liver Disease. Springer, Cham: Springer, 2016.

10. Younossi ZM, Koenig AB, Abdelatif D, et al. Global epidemiology of nonalcoholic fatty liver disease-Metaanalytic assessment of prevalence, incidence, and outcomes. Hepatology 2016;64:73-84.

11. Younossi ZM, Stepanova M, Afendy M, et al. Changes in the prevalence of the most common causes of chronic liver diseases in the United States from 1988 to 2008. Clin Gastroenterol Hepatol 2011;9:524-30.e1; quiz e60.

12. Seto WK, Yuen MF. Nonalcoholic fatty liver disease in Asia: emerging perspectives. J Gastroenterol 2017;52:164-74.

13. Estes C, Razavi H, Loomba R, et al. Modeling the epidemic of nonalcoholic fatty liver disease demonstrates an exponential increase in burden of disease. Hepatology 2018;67:123-33.

14. Seydel GS, Kucukoglu O, Altinbas A, et al. Economic growth leads to increase of obesity and associated hepatocellular carcinoma in developing countries. Ann Hepatol 2016;15:662-72.

15. Perry RJ, Samuel VT, Petersen KF, et al. The role of hepatic lipids in hepatic insulin resistance and type 2 diabetes. Nature 2014;510:84-91.

16. Diehl AM, Day C. Cause, pathogenesis, and treatment of nonalcoholic steatohepatitis. $\mathrm{N}$ Engl J Med 2017;377:2063-72.

17. Byrne CD, Targher G. NAFLD: a multisystem disease. J Hepatol 2015;62:S47-64.

18. Lieber CS, Jones DP, Decarli LM. Effects of prolonged ethanol intake: production of fatty liver despite adequate diets. J Clin Invest 1965;44:1009-21.

19. Méndez-Sánchez N, Almeda-Valdés P, Uribe M. Alcoholic liver disease. An update. Ann Hepatol 2005;4:32-42.

20. GBD 2016 Alcohol and Drug Use Collaborators. The global burden of disease attributable to alcohol and drug use in 195 countries and territories, 1990-2016: a 
systematic analysis for the Global Burden of Disease Study 2016. Lancet Psychiatry 2018;5:987-1012.

21. Loomba R, Sanyal AJ. The global NAFLD epidemic. Nat Rev Gastroenterol Hepatol 2013;10:686-90.

22. Adeyi O, Smith O, Robles S. Public policy and the challenge of chronic non-communicable diseases. Washington, DC: World Bank, 2007.

23. Abegunde DO, Mathers CD, Adam T, et al. The burden and costs of chronic diseases in low-income and middleincome countries. Lancet 2007;370:1929-38.

24. Chalasani N, Younossi Z, Lavine JE, et al. The Diagnosis and Management of Nonalcoholic Fatty Liver Disease: Practice Guidance From the American Association for the Study of Liver Diseases. Hepatology 2018;67:328-57.

25. Rinella ME. Nonalcoholic fatty liver disease. JAMA 2015;313:2263-73.

26. Brunt EM. Non-alcoholic fatty liver disease: what's new under the microscope? Gut 2011;60:1152-8.

27. Adams LA, Lymp JF, St Sauver J, et al. The natural history of nonalcoholic fatty liver disease: a population-based cohort study. Gastroenterology 2005;129:113-21.

28. Harmon RC, Tiniakos DG, Argo CK. Inflammation in nonalcoholic steatohepatitis. Expert Rev Gastroenterol Hepatol 2011;5:189-200.

29. Ekstedt, M, Hagstrom H, Nasr P, et al. Fibrosis stage is the strongest predictor for disease-specific mortality in NAFLD after up to 33 years of follow-up. Hepatology 2015;61:1547-54.

30. Younossi ZM, Stepanova M, Rafiq N, et al. Nonalcoholic Steatofibrosis Independently Predicts Mortality in Nonalcoholic Fatty Liver Disease. Hepatol Commun 2017;1:421-8.

31. Stepanova M, Rafiq N, Makhlouf H, et al. Predictors of all-cause mortality and liver-related mortality in patients with nonalcoholic fatty liver disease (NAFLD). Dig Dis Sci 2013;58:3017-23.

32. Matteoni CA, Younossi ZM, Gramlich T, et al. Nonalcoholic fatty liver disease: a spectrum of clinical and pathological severity. Gastroenterology 1999;116:1413-19.

33. Wong RJ, Aguilar M, Cheung R, et al. Nonalcoholic steatohepatitis is the second leading etiology of liver disease among adults awaiting liver transplantation in the United States. Gastroenterology 2015;148:547-55.

34. Barik A, Shah RV, Spilahari A et al. Hepatic steatosis is associated with cardiometabolic risk in a rural Indian population: A prospective cohort study. Int J Cardiol 2016;225:161-6.

35. Younossi Z, Anstee QM, Marietti M, et al. Global burden of NAFLD and NASH: trends, predictions, risk factors and prevention. Nat Rev Gastroenterol Hepatol 2018;15:11-20.

36. Vernon G, Baranova A, Younossi ZM. Systematic review: the epidemiology and natural history of non-alcoholic fatty liver disease and non-alcoholic steatohepatitis in adults. Aliment Pharmacol Ther 2011;34:274-85.

37. Kleiner DE, Brunt EM. Nonalcoholic fatty liver disease: pathologic patterns and biopsy evaluation in clinical research. Semin Liver Dis 2012;32:3-13.

38. Noureddin M, Lam J, Peterson MR, et al. Utility of magnetic resonance imaging versus histology for quantifying changes in liver fat in nonalcoholic fatty liver disease trials. Hepatology 2013;58:1930-40.

39. Loomba R. Role of imaging-based biomarkers in NAFLD: recent advances in clinical application and future research directions. J Hepatol 2018;68:296-304.

40. Wong VW, Chu WC, Wong GL, et al. Prevalence of nonalcoholic fatty liver disease and advanced fibrosis in Hong Kong Chinese: a population study using proton-magnetic resonance spectroscopy and transient elastography. Gut 2012;61:409-15.

41. Sayiner M, Koenig A, Henry L, et al. Epidemiology of nonalcoholic fatty liver disease and nonalcoholic steatohepatitis in the united states and the rest of the world. Clin Liver Dis 2016;20:205-14.

42. Sherif ZA, Saeed A, Ghavimi S, et al. Global epidemiology of nonalcoholic fatty liver disease and perspectives on US minority populations. Dig Dis Sci 2016;61:1214-25.

43. Wong MCS, Huang JLW, George J, et al. The changing epidemiology of liver diseases in the Asia-Pacific region. Nat Rev Gastroenterol Hepatol 2019;16:57-73.

44. Fan JG, Kim SU, Wong VW. New trends on obesity and NAFLD in Asia. J Hepatol 2017;67:862-73.

45. Li J, Zou B, Yeo YH, et al. Prevalence, incidence, and outcome of non-alcoholic fatty liver disease in Asia, 19992019: a systematic review and meta-analysis. Lancet Gastroenterol Hepatol 2019;4:389-98.

46. Fan J.G. Epidemiology of alcoholic and nonalcoholic fatty liver disease in China. J Gastroenterol Hepatol 2013;28:11-7.

47. Kojima S, Watanabe N, Numata $M$, et al. Increase in the prevalence of fatty liver in Japan over the past 12 years: analysis of clinical background. J Gastroenterol 2003;38:954-61.

48. Park SH, Jeon WK, Kim SH, et al. Prevalence and risk factors of non-alcoholic fatty liver disease among Korean adults. J Gastroenterol Hepatol 2006;21:138-43. 
49. Jeong EH, Jun DW, Cho YK, et al. Regional prevalence of non-alcoholic fatty liver disease in Seoul and Gyeonggido, Korea. Clin Mol Hepatol 2013;19:266-72.

50. Shen L, Fan JG, Shao Y, et al. Prevalence of nonalcoholic fatty liver among administrative officers in Shanghai: an epidemiological survey. World J Gastroenterol 2003;9:1106-10.

51. Zhai HL, Wang NJ, Han B, et al. Low vitamin D levels and non-alcoholic fatty liver disease, evidence for their independent association in men in East China: a crosssectional study (Survey on Prevalence in East China for Metabolic Diseases and Risk Factors (SPECT-China)). Br J Nutr 2016;115:1352-9.

52. Das K, Das K, Mukherjee PS, et al. Nonobese population in a developing country has a high prevalence of nonalcoholic fatty liver and significant liver disease. Hepatology 2010;51:1593-602.

53. Das K, Chowdhury A. Lean NASH: distinctiveness and clinical implication. Hepatol Int 2013;7 Suppl 2:806-13.

54. Zelber-Sagi S, Lotan R, Shlomai A, et al. Predictors for incidence and remission of NAFLD in the general population during a seven-year prospective follow-up. J Hepatol 2012;56:1145-51.

55. Wong VW, Wong GL, Yeung DK, et al. Incidence of nonalcoholic fatty liver disease in Hong Kong: a population study with paired proton-magnetic resonance spectroscopy. J Hepatol 2015;62:182-9.

56. Sung KC, Wild SH, Byrne CD. Development of new fatty liver, or resolution of existing fatty liver, over five years of follow-up, and risk of incident hypertension. J Hepatol 2014;60:1040-5.

57. Kanwal F, Kramer JR, Duan J, et al. Trends in the burden of nonalcoholic fatty liver disease in a United States cohort of veterans. Clin Gastroenterol Hepatol 2016;14:301-8.e1.

58. Bedogni G, Miglioli L, Masutti F, et al. Incidence and natural course of fatty liver in the general population: the Dionysos study. Hepatology 2007;46:1387-91.

59. Ng M, Fleming T, Robinson M, et al. Global, regional, and national prevalence of overweight and obesity in children and adults during 1980-2013: a systematic analysis for the Global Burden of Disease Study 2013. Lancet 2014;384:766-81.

60. Subichin M, Clanton J, Makuszewski M, et al. Liver disease in the morbidly obese: a review of 1000 consecutive patients undergoing weight loss surgery. Surg Obes Relat Dis 2015;11:137-41.

61. Masuoka HC, Chalasani N. Nonalcoholic fatty liver disease: an emerging threat to obese and diabetic individuals. Ann N Y Acad Sci 2013;1281:106-22.

62. Masood M, Reidpath DD. Effect of national wealth on BMI: An analysis of 206,266 individuals in 70 low, middle- and high-income countries. PLoS One 2017;12:e0178928.

63. NCD Risk Factor Collaboration (NCD-RisC). Worldwide trends in body-mass index, underweight, overweight, and obesity from 1975 to 2016: a pooled analysis of 2416 population-based measurement studies in 128.9 million children, adolescents, and adults. Lancet 2017;390:2627-42.

64. Leite NC, Salles GF, Araujo AL, et al. Prevalence and associated factors of non-alcoholic fatty liver disease in patients with type-2 diabetes mellitus. Liver Int 2009;29:113-19.

65. Bril F, Cusi K. Nonalcoholic fatty liver disease: the new complication of type 2 diabetes mellitus. Endocrinol Metab Clin North Am 2016;45:765-81.

66. Fruci B, Giuliano S, Mazza A, et al. Nonalcoholic fatty liver: a possible new target for type 2 diabetes prevention and treatment. Int J Mol Sci 2013;14:22933-66.

67. Goessling W, Massaro JM, Vasan RS, et al. Aminotransferase levels and 20 year risk of metabolic syndrome, diabetes, and cardiovascular disease. Gastroenterology 2008;135:1935-44.

68. Fukuda T, Hamaguchi M, Kojima T, et al. The impact of non-alcoholic fatty liver disease on incident type 2 diabetes mellitus in non-overweight individuals. Liver Int 2016;36:275-83.

69. Sung KC, Wild SH, Byrne CD. Resolution of fatty liver and risk of incident diabetes. J Clin Endocrinol Metab 2013;98:3637-43.

70. Tilg H, Moschen AR, Roden M. NAFLD and diabetes mellitus. Nat Rev Gastroenterol Hepatol 2017;14:32-42.

71. Jimba S, Nakagami T, Takahashi M, et al. Prevalence of non-alcoholic fatty liver disease and its association with impaired glucose metabolism in Japanese adults. Diabet Med 2005;22:1141-5.

72. Fan JG, Zhu J, Li XJ, et al. Prevalence of and risk factors for fatty liver in a general population of Shanghai, China. J Hepatol 2005;43:508-14.

73. Frith J, Day CP, Henderson E, et al. Non-alcoholic fatty liver disease in older people. Gerontology 2009;55:607-13.

74. Kennedy BK, Berger SL, Brunet A, et al. Geroscience: linking aging to chronic disease. Cell 2014;159:709-13.

75. Omagari K, Kadokawa Y, Masuda J, et al. Fatty liver in non-alcoholic non-overweight Japanese adults: incidence and clinical characteristics. J Gastroenterol Hepatol 
2002; 17:1098-105.

76. Chen ZW, Chen LY, Dai HL, et al. Relationship between alanine aminotransferase levels and metabolic syndrome in nonalcoholic fatty liver disease. J Zhejiang Univ Sci B 2008;9:616-22.

77. Papatheodoridis GV, Goulis J, Christodoulou D, et al. High prevalence of elevated liver enzymes in blood donors: associations with male gender and central adiposity. Eur J Gastroenterol Hepatol 2007;19:281-7.

78. Ong JP, Elariny H, Collantes R, et al. Predictors of nonalcoholic steatohepatitis and advanced fibrosis in morbidly obese patients. Obes Surg 2005;15:310-5.

79. Pan JJ, Fallon MB. Gender and racial differences in nonalcoholic fatty liver disease. World J Hepatol 2014;6:274-283.

80. Browning JD, Szczepaniak LS, Dobbins R, et al. Prevalence of hepatic steatosis in an urban population in the United States: impact of ethnicity. Hepatology 2004;40:1387-95.

81. Saab S, Manne V, Nieto J, et al. Nonalcoholic fatty liver disease in Latinos. Clin Gastroenterol Hepatol 2016;14:5-12.

82. Balakrishnan M, Kanwal F, El Serag HB, et al. Acculturation and nonalcoholic fatty liver disease risk among hispanics of mexican origin: findings from the National Health and Nutrition Examination Survey. Clin Gastroenterol Hepatol 2017;15:310-2.

83. Fleischman MW, Budoff $M$, Zeb I, et al. NAFLD prevalence differs among Hispanic subgroups: the MultiEthnic Study of Atherosclerosis. World J Gastroenterol 2014;20: 4987-93.

84. Zimmermann E, Gamborg M, Holst C, et al. Body mass index in school aged children and the risk of routinely diagnosed nonalcoholic fatty liver disease in adulthood: a prospective study based on the Copenhagen School Health Records Register. BMJ Open 2015;5:e006998.

85. Schwimmer JB, Deutsch R, Kahen T, et al. Prevalence of fatty liver in children and adolescents. Pediatrics 2006;118:1388-93.

86. Anderson EL, Howe LD, Jones HE, et al. The prevalence of non-alcoholic fatty liver disease in children and adolescents: a systematic review and meta-analysis. PLoS One 2015;10:e0140908

87. Suomela E, Oikonen M, Pitkänen N, et al. Childhood predictors of adult fatty liver. The Cardiovascular Risk Young Finns Study. J Hepatol 2016;65:784-90.

88. Wesolowski SR, Kasmi KC, Jonscher KR, et al. Developmental origins of NAFLD: a womb with a clue.
Nat Rev Gastroenterol Hepatol 2017;14:81-96.

89. Ayonrinde OT, Adams LA, Mori TA. Sex Differences Between Parental Pregnancy Characteristics and Nonalcoholic Fatty Liver Disease in Adolescents. Hepatology 2018;67:108-22.

90. Kwon YM, Oh SW, Hwang SS, et al. Association of nonalcoholic fatty liver disease with components of metabolic syndrome according to body mass index in Korean adults. Am J Gastroenterol 2012;107:1852-8.

91. Younossi ZM, Stepanova M, Negro F, et al. Nonalcoholic fatty liver disease in lean individuals in the United States. Medicine (Baltimore) 2012;91:319-27.

92. Sinn DH, Gwak GY, Park HN, et al. Ultrasonographically detected non-alcoholic fatty liver disease is an independent predictor for identifying patients with insulin resistance in nonobese, non-diabetic middle-aged Asian adults. Am J Gastroenterol 2012;107:561-7.

93. $\mathrm{Xu} \mathrm{C}, \mathrm{Yu} \mathrm{C}, \mathrm{Ma} \mathrm{H}$, et al. Prevalence and risk factors for the development of nonalcoholic fatty liver disease in a non-obese Chinese population: the Zhejiang Zhenhai Study. Am J Gastroenterol 2013;108:1299-304.

94. Wei JL, Leung JC, Loong TC, et al. Prevalence and severity of nonalcoholic fatty liver disease in nonobese patients: a population study using protonmagnetic resonance spectroscopy. Am J Gastroenterol 2015;110:1306-14.

95. Hagström H, Nasr P, Ekstedt M, et al. Risk for development of severe liver disease in lean patients with nonalcoholic fatty liver disease: a long-term follow-up study. Hepatol Commun 2017;2:48-57.

96. Yoshitaka H, Hamaguchi M, Kojima T, et al. No overweight nonalcoholic fatty liver disease and incident cardiovascular disease: A post hoc analysis of a cohort study. Medicine (Baltimore) 2017;96:e6712.

97. Singh P, Das K, Misra D, et al. Dynamicity of Adiposity determine evolution of non-alcoholic fatty liver even in lean subjects: a prospective cohort study. Hepatology 2014; 60:600A.

98. GBD 2016 Alcohol Collaborators. Alcohol use and burden for 195 countries and territories, 1990-2016: a systematic analysis for the Global Burden of Disease Study 2016. Lancet 2018;392:1015-35.

99. WHO. Global status report on alcohol and health 2018. Geneva: World Health Organization, 2018.

100. Peery AF, Crockett SD, Barritt AS, et al. Burden of Gastrointestinal, Liver, and Pancreatic Diseases in the United States. Gastroenterology 2015;149:1731-41.e3. 101. Sheron N. Alcohol and liver disease in Europe--Simple 
measures have the potential to prevent tens of thousands of premature deaths. J Hepatol 2016;64:957-67.

102. Mukherjee PS, Vishnubhatla S, Amarapurkar DN, et al. Etiology and mode of presentation of chronic liver diseases in India: A multi centric study. PLoS One 2017;12:e0187033.

103.Bell G, Cremona A. Alcohol and death certification: a survey of current practice and attitudes. BMJ 1987;295:95.

104. Alavi M, Janjua NZ, Chong M, et al. The contribution of alcohol use disorder to decompensated cirrhosis among people with hepatitis C: An international study. J Hepatol 2018;68:393-401.

105. Dam-Larsen S. Long term prognosis of fatty liver: risk of chronic liver disease and death. Gut 2004;53:750-5.

106. Lefkowitch JH. Morphology of Alcoholic Liver Disease. Clin Liver Dis 2005;9:37-53.

107.Parker R, Aithal GP, Becker U, et al. Natural history of histologically proven alcohol-related liver disease: A systematic review. J Hepatol 2019;71:586-93.

108. Hughes E, Hopkins LJ, Parker R. Survival from alcoholic hepatitis has not improved over time. PLoS One 2018;13:e0192393.

109. Sandahl TD, Jepsen P, Thomsen KL, et al. Incidence and mortality of alcoholic hepatitis in Denmark 1999-2008: A nationwide population based cohort study. J Hepatol 2011;54:760-4.

110.Sahlman P, Nissinen M, Pukkala E, et al. Incidence, survival and cause-specific mortality in alcoholic liver disease: a population-based cohort study. Scand J Gastroenterol 2016;51:961-6.

111. Chirapongsathorn S, Krittanawong C, Enders FT et al. Incidence and cost analysis of hospital admission and 30day readmission among patients with cirrhosis. Hepatol Commun 2018;2:188-98.

112. Tuyns AJ, Pequingot G. Greater Risk of Ascitic Cirrhosis in Females in Relation to Alcohol Consumption. Int J Epidemiol 1984;13:53-7.

113. Corrao G, Bagnardi V, Zambon A, et al. Exploring the dose-response relationship between alcohol consumption and the risk of several alcohol-related conditions: a metaanalysis. Addiction 1999;94:1551-73.

114. Bellentani S, Saccoccio G, Costa G, et al. Drinking habits as cofactors of risk for alcohol induced liver damage. The Dionysos Study Group. Gut 1997;41:845-50.

115. Becker U, Deis A, Sørensen TI, et al. Prediction of risk of liver disease by alcohol intake, sex, and age: a prospective population study. Hepatology 1996;23:1025-9.

116. Becker U. Lower risk for alcohol-induced cirrhosis in wine drinkers. Hepatology 2002;35:868-75.

117.Simpson RF, Hermon C, Liu B, et al. Alcohol drinking patterns and liver cirrhosis risk: analysis of the prospective UK Million Women Study. Lancet Public Health 2019;4:e41-8.

118. Askgaard G, Grønbæk M, Kjær MS, et al. Alcohol drinking pattern and risk of alcoholic liver cirrhosis: A prospective cohort study. J Hepatol 2015;62:1061-7.

119. Åberg F, Helenius-Hietala J, Puukka P, et al. Binge drinking and the risk of liver events: A population-based cohort study. Liver Int 2017;37:1373-81.

120. Tsukamoto H, Cheng S, Blaner WS. Effects of dietary polyunsaturated fat on ethanol-induced Ito cell activation. Am J Physiol 1996;270:G581-6.

121. Enomoto N, Yamashina S, Schemmer P, et al. Estriol sensitizes rat Kupffer cells via gut-derived endotoxin. Am J Physiol 1999;277:G671-7.

122. Pessione F, Ramond MJ, Peters L, et al. Five-year survival predictive factors in patients with excessive alcohol intake and cirrhosis. Effect of alcoholic hepatitis, smoking and abstinence. Liver Int 2003;23:45-53.

123. Yamanaka T, Shiraki K, Nakazaawa S, et al. Impact of hepatitis $\mathrm{B}$ and $\mathrm{C}$ virus infection on the clinical prognosis of alcoholic liver cirrhosis. Anticancer Res. 2001;21:2937-40.

124. Chen CC, Kuo CJ, Tsai SY, et al. Relation of genotypes of alcohol metabolizing enzymes and mortality of liver diseases in patients with alcohol dependence. Addict Biol 2004;9:233-7.

125. Sánchez-Jiménez BA, Brizuela-Alcántara D, Ramos-Ostos $\mathrm{MH}$, et al. Both alcoholic and non-alcoholic steatohepatitis association with cardiovascular risk and liver fibrosis. Alcohol 2018;69:63-7.

126. Williams R, Taylor-Robinson SD. Clinical dilemmas in non-alcoholic fatty liver disease. Chichester, UK: John Wiley \& Sons 2016.

127. Brunt EM. What's in a Name? Hepatology 2009;50:663-7.

128. Bellentani S, Saccoccio G, Masutti F, et al. Prevalence of and Risk Factors for Hepatic Steatosis in Northern Italy. Ann Intern Med 2000;132:112.

129. Moriya A, Iwasaki Y, Ohguchi S, et al. Alcohol consumption appears to protect against non-alcoholic fatty liver disease. Aliment Pharmacol Ther 2011;33:378-88.

130. Sookoian S, Castaño GO, Pirola CJ. Modest alcohol consumption decreases the risk of non-alcoholic fatty liver disease: a meta-analysis of 43175 individuals. Gut 2014;63:530-2.

131.Hamaguchi M, Kojima T, Ohbora A, et al. Protective 
effect of alcohol consumption for fatty liver but not metabolic syndrome. World J Gastroenterol 2012;18:156-67.

132. Takahashi H, Ono M, Hyogo H, et al. Biphasic effect of alcohol intake on the development of fatty liver disease. J Gastroenterol 2015;50:1114-23.

133. Hiramine Y, Imamura Y, Uto H, et al. Alcohol drinking patterns and the risk of fatty liver in Japanese men. J Gastroenterol 2011;46:519-28.

134. Gunji T, Matsuhashi N, Sato H, et al. Light and Moderate Alcohol Consumption Significantly Reduces the Prevalence of Fatty Liver in the Japanese Male Population. Am J Gastroenterol 2009;104:2189-95.

135. Sogabe M, Okahisa T, Taniguchi T, et al. Light alcohol consumption plays a protective role against non-alcoholic fatty liver disease in Japanese men with metabolic syndrome. Liver Int 2015;35:1707-14.

136. Dunn W, Xu R, Schwimmer JB. Modest wine drinking and decreased prevalence of suspected nonalcoholic fatty liver disease. Hepatology 2008;47:1947-54.

137. Dunn W, Sanyal AJ, Brunt EM, et al. Modest alcohol consumption is associated with decreased prevalence of steatohepatitis in patients with non-alcoholic fatty liver disease (NAFLD). J Hepatol 2012;57:384-91.

138. Ekstedt M, Franzén LE, Holmqvist M, et al. Alcohol consumption is associated with progression of hepatic fibrosis in non-alcoholic fatty liver disease. Scand J Gastroenterol 2009;44:366-74.

139. Sookoian S, Pirola CJ. How Safe Is Moderate Alcohol Consumption in Overweight and Obese Individuals? Gastroenterology 2016;150:1698-703.e2.

140.Ajmera V, Belt P, Wilson LA, et al. Among Patients With Nonalcoholic Fatty Liver Disease, Modest Alcohol Use Is Associated With Less Improvement in Histologic Steatosis and Steatohepatitis. Clin Gastroenterol Hepatol 2018;16:1511-20.e5.

141.Chang Y, Cho YK, Kim Y, et al. Nonheavy drinking and worsening of noninvasive fibrosis markers in nonalcoholic fatty liver disease: a cohort study. Hepatology 2019;69:64-75.

142. Mehta M, Satsangi S, Duseja A et al. Can Alcoholic Liver Disease and Nonalcoholic Fatty Liver Disease Co-Exist? J Clin Exp Hepatol 2017;7:121-6.

143. Duseja AK, De A, Taneja S, et al. Prevalence of Metabolic Risk Factors and Its Impact on the Severity and Outcome of Patients with Alcohol Related Acute-on-Chronic-Liver Failure (ACLF) - a Case Control Study from the APASL ACLF Research Consortium (AARC) Data Base. San
Francisco: AASLD, 2018.

144. Chamorro AJ, Torres JL, Mirón-Canelo JA, et al Systematic review with meta-analysis: the I148M variant of patatin-like phospholipase domain-containing 3 gene (PNPLA3) is significantly associated with alcoholic liver cirrhosis. Aliment Pharmacol Ther 2014;40:571-81.

145. Fattovich G, Stroffolini T, Zagni I, et al. Hepatocellular carcinoma in cirrhosis: incidence and risk factors. Gastroenterology 2004;127:S35-50.

146. Ganne-Carrié N, Chaffaut C, Bourcier V, et al. Estimate of hepatocellular carcinoma incidence in patients with alcoholic cirrhosis. J Hepatol 2018;69:1274-83.

147.European Association for the Study of the Liver. EASL Clinical Practice Guidelines: Liver transplantation. J Hepatol 2016;64:433-85.

148. Bachir NM, Larson AM, Palmer BF. Adult Liver Transplantation in the United States. Am J Med Sci 2012;343:462-9.

149.Lucey MR. Liver transplantation for alcoholic liver disease. Nat Rev Gastroenterol Hepatol 2014;11:300-7.

150.Lee BP, Vittinghoff E, Hsu C, et al. Predicting Low Risk for Sustained Alcohol Use After Early Liver Transplant for Acute Alcoholic Hepatitis: The Sustained Alcohol Use Post-Liver Transplant Score. Hepatology 2019;69:1477-87.

151. Mathurin P, Moreno C, Samuel D, et al. Early Liver Transplantation for Severe Alcoholic Hepatitis. N Engl J Med 2011;365:1790-800.

152.Lee BP, Chen PH, Haugen C, et al. Three-year Results of a Pilot Program in Early Liver Transplantation for Severe Alcoholic Hepatitis. Ann Surg 2017;265:20-9.

153.Im GY, Kim-Schluger L, Shenoy A, et al. Early Liver Transplantation for Severe Alcoholic Hepatitis in the United States--A Single-Center Experience. Am J Transplant 2016;16:841-9.

154. Singal AK, Anand BS. Recent trends in the epidemiology of alcoholic liver disease. Clin Liver Dis (Hoboken) 2013;2:53-6.

155. Bush K, Kivlahan DR, McDonell MB, et al. The AUDIT alcohol consumption questions (AUDIT-C): an effective brief screening test for problem drinking. Ambulatory Care Quality Improvement Project (ACQUIP). Alcohol Use Disorders Identification Test. Arch Intern Med 1998;158:1789.

156. Crabb DW, Im GY, Szabo G, et al. Diagnosis and Treatment of Alcohol-Related Liver Diseases: 2019 Practice Guidance from the American Association for the Study of Liver Diseases. Hepatology 2019. [Epub ahead of 
print].

157. Department of Health. UK Chief Medical Officers' Low Risk Drinking Guidelines 2016. Available online: www.drinkaware.co.uk

doi: $10.21037 / \operatorname{tgh} .2019 .09 .08$

Cite this article as: Mitra S, De A, Chowdhury A. Epidemiology of non-alcoholic and alcoholic fatty liver diseases. Transl Gastroenterol Hepatol 2020;5:16.
158. European Association for the Study of the Liver. EASL Clinical Practice Guidelines: Management of alcoholrelated liver disease. J Hepatol 2018;69:154-81. 